\title{
The role of arginase $I$ in diabetes-induced retinal vascular dysfunction in mouse and rat models of diabetes
}

\author{
S. C. Elms • H. A. Toque $\cdot$ M. Rojas $\cdot$ Z. Xu • \\ R. W. Caldwell $\cdot$ R. B. Caldwell
}

Received: 20 September 2012 / Accepted: 7 November 2012 /Published online: 12 December 2012

(C) Springer-Verlag Berlin Heidelberg 2012

\begin{abstract}
Aims/hypothesis A reduction in retinal blood flow occurs early in diabetes and is likely to be involved in the development of diabetic retinopathy. We hypothesise that activation of the arginase pathway could have a role in the vascular dysfunction of diabetic retinopathy.

Methods Experiments were performed using a mouse and rat model of streptozotocin (STZ)-induced diabetes for in vivo and ex vivo analysis of retinal vascular function. For in vivo studies, mice were infused with the endothelialdependent vasodilator acetylcholine (ACh) or the endothelialindependent vasodilator sodium nitroprusside (SNP), and vasodilation was assessed using a fundus microscope. Ex vivo assays included pressurised vessel myography, western blotting and arginase activity measurements.

Results ACh-induced retinal vasodilation was markedly impaired in diabetic mice ( $40 \%$ of control values), whereas SNP-induced dilation was not altered. The diabetes-induced vascular dysfunction was markedly blunted in mice lacking one copy of the gene encoding arginase I and in mice treated with the arginase inhibitor 2(S)-amino-6-boronohexanoic acid. Ex vivo studies performed using pressure myography and central retinal arteries isolated from rats with STZ-induced diabetes showed a similar impairment of endothelial-dependent vasodilation that was partially blunted
\end{abstract}

S. C. Elms $\cdot$ M. Rojas $\cdot$ Z. Xu $\cdot$ R. B. Caldwell $(\bowtie)$

Vascular Biology Center, Georgia Health Sciences University,

1459 Laney Walker Boulevard,

Augusta, GA 30912, USA

e-mail: rcaldwel@georgiahealth.edu

H. A. Toque $\cdot$ R. W. Caldwell

Department of Pharmacology and Toxicology,

Georgia Health Sciences University, Augusta, GA, USA

R. B. Caldwell

Charlie Norwood VA Medical Center, Augusta, GA, USA by pretreatment of the isolated vessels with another arginase inhibitor, (S)-2-boronoethyl-L-cysteine. The diabetes-induced vascular alterations were associated with significant increases in both arginase I protein levels and total arginase activity. Conclusions/interpretation These results indicate that, in the mouse and rat model, diabetes-induced increases in arginase I were involved in the diabetes-induced impairment of retinal blood flow by a mechanism involving vascular endothelial cell dysfunction.

Keywords Arginase - Endothelium · Fundus imaging · Nitric oxide $\cdot$ Retinopathy $\cdot$ Vascular dysfunction

$\begin{array}{ll}\text { Abbreviations } \\ \text { ABH } & \text { 2(S)-Amino-6-boronohexanoic acid } \\ \text { ACh } & \text { Acetylcholine } \\ \text { BEC } & \text { (S)-2-Boronoethyl-L-cysteine } \\ \text { CRA } & \text { Central retinal artery } \\ \text { DR } & \text { Diabetic retinopathy } \\ \text { EC } & \text { Endothelial cell } \\ \text { eNOS } & \text { Endothelial nitric oxide synthase } \\ \text { HR } & \text { Heart rate } \\ \text { MAP } & \text { Mean arterial pressure } \\ \text { L-NAME } & \text { L-NG-nitroarginine methyl ester } \\ \text { NO } & \text { Nitric oxide } \\ \text { PKC } & \text { Protein kinase C } \\ \text { PSS } & \text { Physiological salt solution } \\ \text { SNP } & \text { Sodium nitroprusside } \\ \text { STZ } & \text { Streptozotocin } \\ \text { WT } & \text { Wild-type }\end{array}$

\section{Introduction}

Diabetic retinopathy (DR) has been shown to be associated with decreases in retinal blood flow in both humans and animal models [1-3]. This is proposed to be mediated by 
numerous mechanisms, but it is very likely that dysfunction of the vascular endothelium is involved.

Vascular ECs contain endothelial nitric oxide synthase (eNOS), an enzyme that hydrolyses L-arginine to form Lcitrulline and NO. NO acts as a major signalling molecule and is required for healthy vascular function [4-6]. NO increases blood flow by activating guanylyl cyclase within the vascular smooth muscle cells, which dilates the vessels. Adequate production of NO by eNOS is necessary for the maintenance of healthy vessels, proper blood flow, prevention of leucocyte adhesion and platelet aggregation, and control of smooth muscle cell growth [5-7]. A decrease in bioavailable NO is a well-established mediator of endothelial cell (EC) dysfunction and is strongly implicated in diabetic vascular disease [8-10]. NOS produces NO from its substrate L-arginine. If the supply of L-arginine is insufficient, uncoupling of the NOS homodimer can occur, causing it to produce superoxide [11]. Superoxide can react with $\mathrm{NO}$ to form peroxynitrite $\left(\mathrm{ONOO}^{-}\right)$, further reducing levels of $\mathrm{NO}$ and increasing oxidative stress [12].

Also residing within ECs is the homotrimer ureahydrolase enzyme arginase. Arginase exists in two isoforms, arginase I and arginase II, that localise to the cytosol and mitochondria, respectively. Both are expressed in ECs. Studies have shown that increases in arginase protein levels and/or activity are associated with vascular dysfunction in diseases such as hypertension, ischaemia reperfusion, ageing and diabetes [13-19]. The role of arginase in DR has not been determined. Excessive arginase activity has been suggested to reduce the production of $\mathrm{NO}$ by reducing the Larginine supply for eNOS [20-22]. Regardless of the mechanism, common pathologies are observed in nearly all conditions of increased arginase activity: redox imbalance, EC dysfunction, reduced blood flow and ischaemia. DR exhibits all of these alterations. Previous work has demonstrated a role for arginase I in diabetes-induced vascular dysfunction of the aorta and coronary vessels, and excessive arginase activity has been implicated in retinal inflammation [18, 23]. Therefore we hypothesised that arginase could be involved in vascular dysfunction associated with DR.

In this study, we tested whether limiting arginase activity could improve blood vessel function in the diabetic retina. This was accomplished using a novel murine fundoscope methodology for in vivo studies in mice and pressure myography for ex vivo studies in rats, as well as arginase activity and protein quantification.

\section{Methods}

Rats and mice Animal procedures were conducted in accordance with the Principles of Laboratory Animal Care (NIH publication no. 85023, revised 1985) and the guidelines of the Institutional Animal Care and Use Committee. Arginase I hemizygous knockout mice $\left(\mathrm{ArgI} \mathrm{I}^{+/}\right)$on a $\mathrm{C} 57 \mathrm{BL} / 6 \mathrm{~J}$ background, C57BL/6J mice and Sprague-Dawley rats were obtained from Jackson Laboratories (Bar Harbor, ME, USA). Male mice were injected intraperitoneally with streptozotocin (STZ, $50 \mathrm{mg} / \mathrm{kg}$ ) on alternating days for up to three injections and were studied after 8 weeks with diabetes. Male rats were injected intraperitoneally with STZ $(65 \mathrm{mg} / \mathrm{kg})$ and were studied after 4 weeks with diabetes.

Arginase activity assay The arginase activity assay is a spectrophotometric measure of urea, one of the two products of arginase activity. Urea is a terminal metabolite and does not undergo further physiological processing in the way that ornithine, the other product of arginase, does. Whole retinas were removed, frozen in liquid nitrogen, crushed and collected in lysis buffer ( $50 \mathrm{mmol} / 1$ Tris-HCl, $0.1 \mathrm{mmol} / \mathrm{l}$ EDTA and EGTA, pH7.5) containing protease inhibitors. Tissues were lysed, subjected to three freeze/thaw cycles and then centrifuged at $20,817 \mathrm{~g}(10 \mathrm{~min})$. The supernatant fraction $(25 \mu \mathrm{l})$ was heated with $25 \mu \mathrm{l} \mathrm{MnCl} 2\left(10 \mathrm{mmol} / \mathrm{l}, 10 \mathrm{~min}, 56^{\circ} \mathrm{C}\right)$ to activate the arginase. The mixture was then incubated with $50 \mu \mathrm{l}$-arginine $\left(0.5 \mathrm{~mol} / \mathrm{l}, \mathrm{pH} 9.7,1 \mathrm{~h}, 37^{\circ} \mathrm{C}\right)$. The hydrolysis reaction was stopped with $400 \mu \mathrm{l}$ acid $\left(\mathrm{H}_{2} \mathrm{SO}_{4}: \mathrm{H}_{3} \mathrm{PO}_{4}\right.$ : $\mathrm{H}_{2} \mathrm{O}$ in a 1:3:7 ratio), and the mixture was heated at $100^{\circ} \mathrm{C}$ with the chromagen $\alpha$-isonitrosopropiophenone ( $9 \%$ in EtOH, $25 \mu \mathrm{l})$ for $45 \mathrm{~min}$. Samples were kept in the dark at room temperature for $10 \mathrm{~min}$, and absorbance was measured at $540 \mathrm{~nm}$. Arginase activity is reported as picomoles of urea per milligram of protein per hour. The remainder of the supernatant fraction was used for western blotting.

Western blotting Retinal homogenates were prepared as described for the arginase activity assay. Proteins were separated on SDS-PAGE and transferred onto nitrocellulose membrane, blocked in 5\% milk Tris-buffered saline with $0.5 \%$ Tween-20. The membrane was incubated overnight $\left(4^{\circ} \mathrm{C}\right)$ in primary antibodies diluted in the blocking solution, washed and incubated in secondary antibodies (conjugated with horseradish peroxidase) for $1 \mathrm{~h}$ at room temperature and developed using chemiluminescence.

Mean arterial blood pressure and heart rate measurements Mice were anaesthetised using an isoflurane vapouriser. The right jugular vein was accessed with a PE-10 catheter for injection of acetylcholine (ACh), sodium nitroprusside (SNP) or vehicle. The left carotid artery was cannulated with a similar catheter connected to a pressure transducer for the direct measurement of mean arterial pressure (MAP) and heart rate (HR). MAP and HR were recorded and analysed with a PowerLab data acquisition system (AD Instruments, Boston, MA, USA). 
Mouse vascular function studies The Micron III (Phoenix Research Laboratories, Pleasanton, CA, USA) retinal imaging system was used to visualise and measure vessel reactivity. The mice were anaesthetised using isoflurane, and ophthalmic gel (hypromellose $2.5 \%$ ophthalmic demulcent solution) was applied to the eye. The jugular vein was cannulated with a polyethylene catheter (PE-10) to inject FITC dextran $(50 \mathrm{mg} / \mathrm{ml}$; Sigma-Aldrich, St Louis, MO, USA), and the retinal vessels were imaged.

The jugular cannula was then switched to a syringe containing either ACh or SNP and attached to a syringe pump (Harvard Apparatus, Holliston, MA, USA). The infusion rate was adjusted to inject each concentration noted over $5 \min \left(0.3,0.5,1,3,5\right.$ and $\left.10 \mu \mathrm{gmin}^{-1} \mathrm{~kg}^{-1}\right)$. Arteries were identified by their pulsatile activity and branch anatomy. A recognisable anatomical point two optic disc diameters from the optic disc was chosen for each mouse. A $10 \mathrm{~s}$ video was captured at the end of each 5 min interval. A pixel count was collected at the same anatomical point from edge to edge of the vessel in question. Each pixel was $2.25 \mu \mathrm{m}$ in size, and the vessel diameter was calculated based on the number of pixels.

Measurements were taken by two independent investigators blinded to the treatment conditions. Variability of measurement between observers was assessed by ANOVA, which showed no significant differences between observers or between treatment groups. Measurements of vasorelaxation are given as the percentage change from baseline (pre-infusion).

Rat vascular function studies Rats were anaesthetised with $100 \mathrm{mg} / \mathrm{kg}$ ketamine- $10 \mathrm{mg} / \mathrm{kg}$ xylazine (i.p.) and the eyeballs were removed. Segments of the central retinal artery (CRA) were isolated under physiological salt solution (PSS; $\mathrm{NaCl} 118 \mathrm{mmol} / \mathrm{l}, \mathrm{NaHCO}_{3} 25 \mathrm{mmol} / \mathrm{l}$, glucose $5.6 \mathrm{mmol} / \mathrm{l}$, $\mathrm{KCl} 4.7 \mathrm{mmol} / 1, \mathrm{KH}_{2} \mathrm{PO}_{4} 1.2 \mathrm{mmol} / \mathrm{l}, \mathrm{MgSO}_{4} 7 \mathrm{H}_{2} \mathrm{O}$ $\left.1.17 \mathrm{mmol} / 1, \mathrm{CaCl}_{2} \cdot 2 \mathrm{H}_{2} \mathrm{O} 2.5 \mathrm{mmol} / \mathrm{l}\right)$.

The CRA (40-60 $\mu \mathrm{m}$ in internal diameter) was carefully cleaned from the connective tissues and transferred to the pressure myograph (Model 111P; Version 3.2, DMT, Aarhus, Denmark), filled with oxygenated PSS at $37^{\circ} \mathrm{C}$. Both ends of the CRA were applied to glass cannulas and tied with nylon 10/0 ophthalmic suture. Each cannula was connected to a reservoir filled with oxygenated physiologic Krebs solution. The pressure of the reservoirs determined the intraluminal pressure. Two pressure transducers measured the inflow and outflow pressures. The internal diameter was monitored continuously by an inverted microscope digital video system. Vesselview software (PhysioLogic, Aarhus, Denmark) was used to calculate the vascular responses.

The response of the vessel segments to changes in transmural pressure was studied without flow. After mounting, the CRAs were equilibrated first at a pressure of $20 \mathrm{mmHg}$ for $30 \mathrm{~min}$ before pressure was gradually increased to
$40 \mathrm{mmHg}$ for an additional $30 \mathrm{~min}$. Thereafter, the vessels were preconstricted with a thromboxane analogue (U-46619, $\left.3 \times 10^{-7} \mathrm{~mol} / \mathrm{l}\right)$. This concentration was chosen based on preliminary experiments showing that it caused a submaximal contraction (about $70 \%$ ). After the vessels had developed a stable basal tone, endothelium-dependent NO-mediated vasodilation to $\mathrm{ACh}\left(10^{-9}-10^{-4} \mathrm{~mol} / \mathrm{l}\right)$ or endothelium-independent vasodilation to SNP $\left(10^{-10}-10^{-5} \mathrm{~mol} / \mathrm{l}\right)$ was obtained.

Involvement of NOS in ACh-induced vasodilation was confirmed using the NOS inhibitor L-NG-nitroarginine methyl ester (L-NAME; $100 \mu \mathrm{mol} / \mathrm{l}$ ). To confirm the role of arginase in diabetic vessels, vessels were pretreated with the arginase inhibitor (S)-2-boronoethyl-L-cysteine (BEC; $100 \mu \mathrm{mol} / \mathrm{l}$, $45 \mathrm{~min}$ ) and concentration-response curves for ACh were constructed in the absence or presence of the inhibitor. At the end of each experiment, the vessel was dilated in calciumfree PSS containing EDTA $(1 \mathrm{mmol} / \mathrm{l})$ to obtain its maximal diameter. All diameter changes in response to agonists were normalised to this maximal vasodilation and expressed as a percentage of maximal dilation.

Statistical analyses The statistical significance of group treatment effects was determined by one-way or two-way ANOVA and post-hoc comparisons. Data are reported as mean \pm SEM.

\section{Results}

Diabetes increases retinal arginase activity and arginase I protein levels in mice Blood glucose level measured at the time the mice were killed was significantly increased in both wild-type (WT) STZ and $\mathrm{ArgI}{ }^{+-}$STZ mice compared with non-diabetic controls $(p<0.05$; Fig. 1a). Diabetes also significantly increased arginase activity in the WT and $\mathrm{ArgI} \mathrm{H}^{+/}$ STZ mice compared with the non-diabetic controls $(p<0.05$; WT STZ, $207 \pm 6 \%$ compared with WT, $100 \pm 2 \%$; and $\mathrm{ArgI}^{+^{\prime-}}$ STZ, $148 \pm 9 \%$ compared with $\mathrm{ArgI}^{\mathrm{H}^{+-}}, 115 \pm 4 \%$ ) (Fig. 1b). Arginase activity was reduced in the $\mathrm{ArgI}^{+/}$STZ compared with the WT STZ group $(p<0.05)$. Western blotting showed that arginase I protein was significantly increased in the WT STZ retinas compared with the WT controls and $\mathrm{ArgI}^{+/}$controls $(p<0.05$; Fig. 1c).

Mouse vascular function studies A representative retinal image and magnification of the image show how the vessel diameter was measured using the Micron III Fundus Microscope (Fig. 2a). Pre-infusion diameters were taken for all four groups, to determine the effect of genotype or diabetes on vessel size. Retinal arteries were selected based on morphology and branching patterns, and the diameter was measured at a location two optic disc diameters from the nerve head. Repeated measurements by two observers showed no 
a
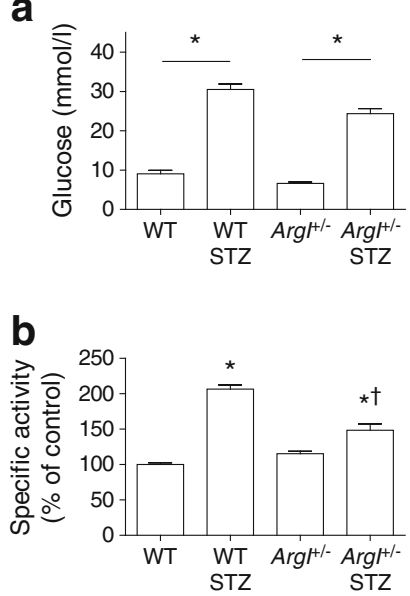

Fig. 1 Blood glucose, arginase activity and arginase I protein levels assessed as described in the Methods section. (a) WT STZ and $\mathrm{ArgI}^{I^{+/}}$ STZ mice had significantly higher blood glucose levels than the nondiabetic controls $\left({ }^{*} p<0.05\right.$ vs all groups, ${ }^{\dagger} p<0.05$ vs WT STZ, oneway ANOVA; WT, $n=9$; WT STZ, $n=6 ; \operatorname{ArgI}^{+/}, n=10 ; \operatorname{ArgI}^{+/}$STZ, $n=6$ ). (b) WT STZ and $\operatorname{Arg} I^{+/}$STZ retinas showed significantly greater arginase activity than those from non-diabetic controls $\left({ }^{*} p<0.05\right.$, oneway ANOVA; all groups, $n=4)$. (c) WT STZ retinas showed higher levels of $\mathrm{ArgI}$ protein compared with WT and $\mathrm{ArgI} \mathrm{I}^{+/}$retinas. Actin protein was used for loading control. $\left({ }^{*} p<0.05\right.$, one-way ANOVA; WT, $n=9$; WT STZ, $\left.n=8 ; \operatorname{ArgI} I^{+/}, n=11 ; \operatorname{ArgI}^{T^{+-}} \mathrm{STZ}, n=4\right)$

significant differences between observers or between the vessel diameters in the different treatment groups (Fig. 2b).

ACh infusion was used to assess endothelium-dependent vasodilation, and SNP was used to assess endotheliumindependent vasodilation. Results are reported as a percentage change from baseline pre-infusion diameter. In WT mice, ACh dilated retinal vessels maximally at the $5 \mu \mathrm{g}$ $\min ^{-1} \mathrm{~kg}^{-1}$ dose $(137 \% \pm 6 \%)$. SNP $\left(10 \mu \mathrm{g} \mathrm{min}^{-1} \mathrm{~kg}^{-1}\right)$ reliably dilated the vessels by $140 \%$ in all groups. As a control to demonstrate the specificity of the ACh-induced dilation, the ACh muscarinic receptor blocker atropine $(0.04 \mathrm{mg} / \mathrm{kg})$,

a
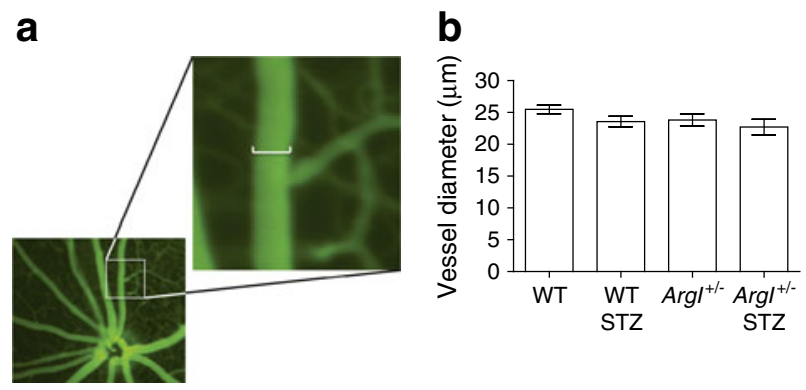

Fig. 2 Diameter of the secondary retinal arterioles measured using the Micron III fundoscope as described in the Methods section. (a) Measurements were taken from fully magnified images in which one pixel $=2.25 \mu \mathrm{m}$. The scale bar in the enlargement represents 12 pixels $(27 \mu \mathrm{m})$. (b) Vessel diameter measurements taken pre-infusion (at baseline) showed no differences between the groups (one-way ANOVA; WT, $n=31$; WT STZ, $n=12 ; \operatorname{ArgI^{+/}}, n=20 ; \operatorname{Arg} I^{+/-}$STZ, $n=16$ ) was administered intravenously prior to ACh infusion. ACh-dependent vasodilation was completely prevented with this pretreatment, but SNP dilation was not significantly changed $(131 \% \pm 1)$. From the $1 \mu \mathrm{gmin}^{-1} \mathrm{~kg}^{-1}$ dose until the final $10 \mu \mathrm{gmin}^{-1} \mathrm{~kg}^{-1}$ dose, the ACh, SNP and SNP + atropine vasodilations were all significantly higher than that seen with $\mathrm{ACh}+$ atropine $(p<0.05$; Fig. 3$)$. Infusion of vehicle alone had no effect on the retinal vessels.

The vascular function analysis showed that vessels from the WT and $\mathrm{ArgI} \mathrm{I}^{+/}$non-diabetic control mice both dilated maximally in a similar manner to ACh and SNP (WT Ach, $142 \pm 2 \%$; WT SNP, $143 \pm 4 \%$; $A r g I^{H^{-}} \mathrm{ACh}, 139 \pm 5 \%, \operatorname{ArgI} I^{+/}$ $\mathrm{SNP}=142 \% \pm 7 \%$ ). Retinal vascular dilation in response to ACh (at 3-10 $\mathrm{gmin}^{-1} \mathrm{~kg}^{-1}$ ) was significantly reduced in the WT STZ mice $(116 \pm 1 \% ; p<0.05)$ compared with WT and $\mathrm{ArgI}^{+/-}$non-diabetic controls. Importantly, the $\mathrm{ArgI}^{+/-} \mathrm{STZ}$ mice had a significantly greater capacity to respond to ACh than the WT STZ mice over the $3-10 \mu \mathrm{gmin}^{-1} \mathrm{~kg}^{-1}$ dose range $(p<0.05)$. SNP-mediated vasodilation was similar in all groups (WT, $143 \pm 4 \%$; $A r g I^{+-}, 142 \pm 7 \%$; WT STZ, $132 \pm 4 \%$; $\mathrm{ArgI}^{+/}$STZ, 129 $\pm 5 \%$ ) (Fig. 4).

In order to assess whether a change in blood pressure or HR could explain the effects of diabetes on retinal vasodilation, we determined the effects of ACh, SNP or vehicle alone on MAP and HR. Both ACh and SNP led to a similar drop in MAP across all four groups (WT, $15 \pm 5 \%$; WT STZ, $8.9 \pm 3 \%$; $\mathrm{ArgI}^{+/-}, 13 \pm 6 \%$; $\operatorname{Arg} \mathrm{I}^{+/} \mathrm{STZ}, 17 \pm 6 \%$ ) (Fig. 5a). Owing to the baroreflex-blocking actions of isoflurane anaesthesia, HR remained constant for the duration of the ACh treatment (Fig. 5b). There were no differences in HR among the four groups. Infusion of vehicle alone had no effect on MAP or HR.

To validate the results seen with partial deletion of the gene encoding arginase I, we treated mice with 2(S)-amino-

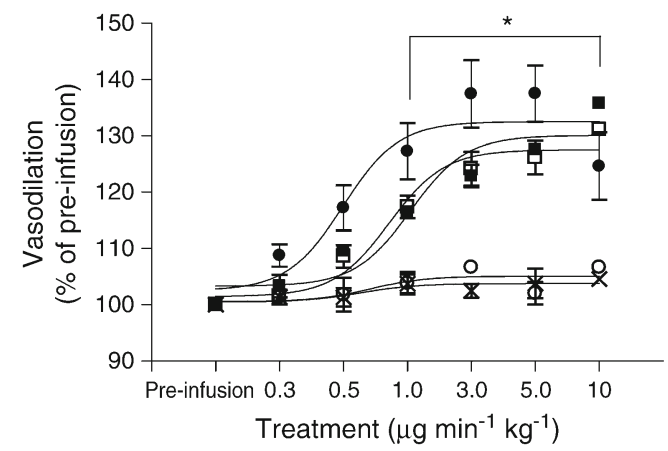

Fig. 3 Fundus imaging of the retinal vasculature in non-diabetic WT mice showed that the retinal vessels dilated equally to SNP and ACh at maximal dilation. Pretreatment with the muscarinic receptor blocker atropine completely blocked dilation to ACh at all doses, but had no effect on SNP-mediated dilation. The vehicle had no effect on retinal vasodilation. $(* p<0.05$ WT ACh + atropine compared with all other groups, two-way ANOVA; all groups, $n=5$ ). Black circle, WT ACh; black square, WT SNP; white circle, WT ACh + atropine; white square, WT SNP + atropine; cross, vehicle 


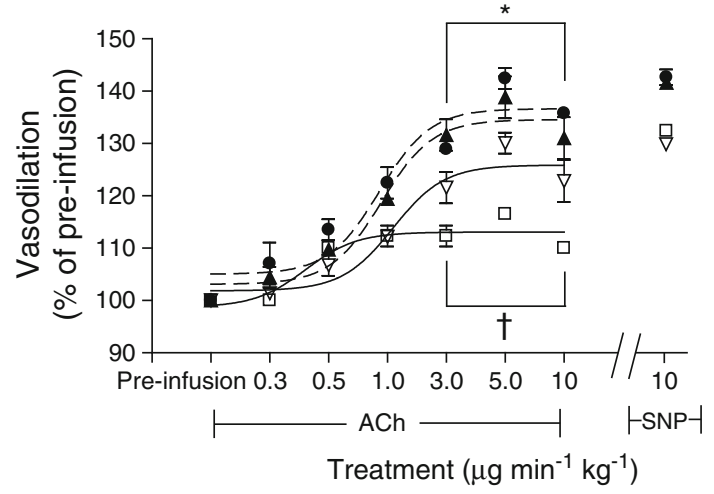

Fig. 4 Fundus imaging of the retinal vasculature of STZ-diabetic and age-matched control WT and $\mathrm{ArgI} \mathrm{I}^{+/}$mice, showing that vasodilation to ACh was markedly reduced in the vessels of WT STZ mice compared with the other three groups. ACh-induced dilation was significantly enhanced in the $\mathrm{ArgI}^{+/} \mathrm{STZ}$ mice compared with the WT STZ mice. All four groups responded similarly to SNP $\left({ }^{*} p<0.05 \mathrm{WT}\right.$ and $\mathrm{ArgI}^{+/}$compared with WT STZ; ${ }^{\dagger} p<0.05 \mathrm{ArgI}^{+/}$STZ compared with WT STZ, two-way ANOVA; WT, WT STZ and $\mathrm{ArgI} I^{+/}, n=4 ; \mathrm{ArgI}^{+/}$ STZ, $n=6$ ). Black circle, WT; white square, WT STZ; black triangle, $\mathrm{ArgI}^{+/-}$; white triangle, $\mathrm{ArgI} \mathrm{H}^{+/-} \mathrm{STZ}$
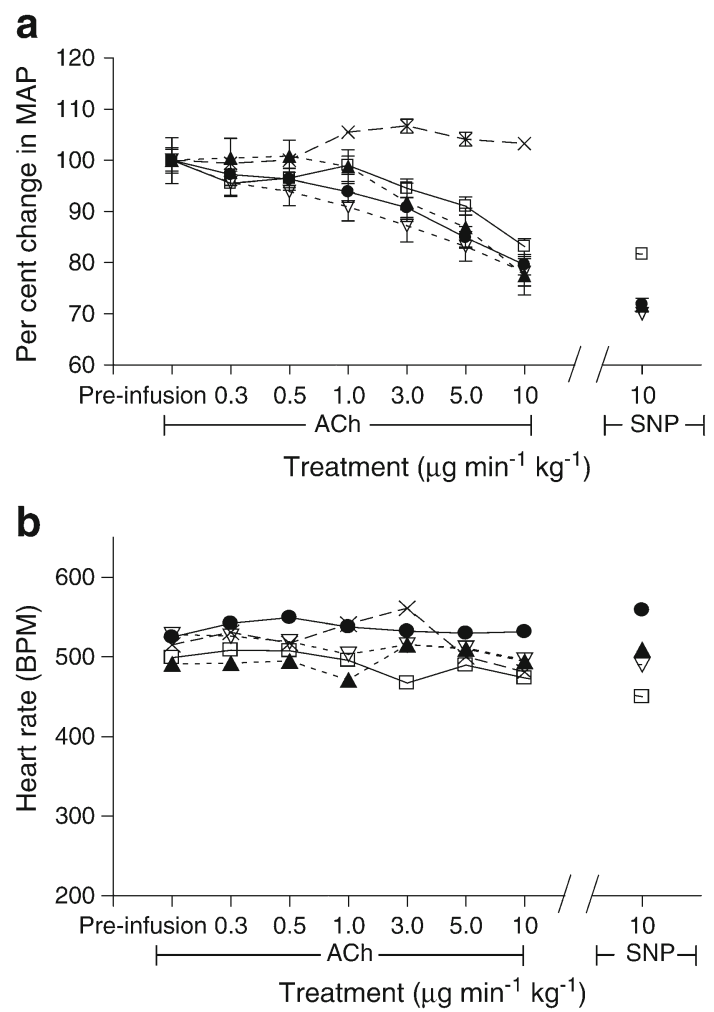

Fig. 5 Blood pressure and HR were not altered by diabetes or heterozygous deletion of arginase I. (a) ACh and SNP elicited equal depressant effects on blood pressure in all groups. (b) ACh and SNP had no effect on HR over the entire course of infusion, and there were no differences between the groups. The vehicle had no effect on either MAP or HR (all groups, $n=4$ ). Black circle, WT; white square, WT STZ; black triangle, $\operatorname{ArgI}^{I^{+-}}$; white triangle, $\operatorname{Arg}^{I^{+-}} \mathrm{STZ}$; cross, vehicle 6-boronohexanoic acid ( $\mathrm{ABH} ; 8 \mathrm{mg} / \mathrm{kg}$ s.c.) for 5 days prior to assessment of retinal vascular function. ABH binds within the arginase homotrimer as a tetrahedral boronate anion and prevents the arginine hydrolysis reaction without affecting eNOS $[24,25]$; it has been shown to be effective in inhibiting arginase activity when given in vivo [26].

The ABH treatment reduced retinal arginase activity to a level comparable with that of the WT and $\mathrm{ArgI}^{+/-}$control groups. As we had previously observed, WT STZ retinas had significantly higher arginase activity than both control WT and $\mathrm{ArgI}^{+/-}$retinas $(p<0.05$; WT STZ, $170 \pm 25 \%$; WT, $100 \pm 8 \% ; \mathrm{ArgI}^{+/-}, 97 \pm 10 \%$ ). Arginase activity in the WT STZ retinas was also significantly higher than in the WT STZ animals treated with ABH $(p<0.05$; WT STZ ABH, $131 \pm 8 \%$ ) (Fig. 6a). The ABH-induced reduction in arginase activity was accompanied by a significant improvement in ACh-mediated endothelium-dependent vasodilation in WT STZ mice compared with untreated WT STZ mice $(p<0.05$ between the 3 and $10 \mu \mathrm{gmin}^{-1} \mathrm{~kg}^{-1}$ doses; Fig. $6 \mathrm{~b}$ ).
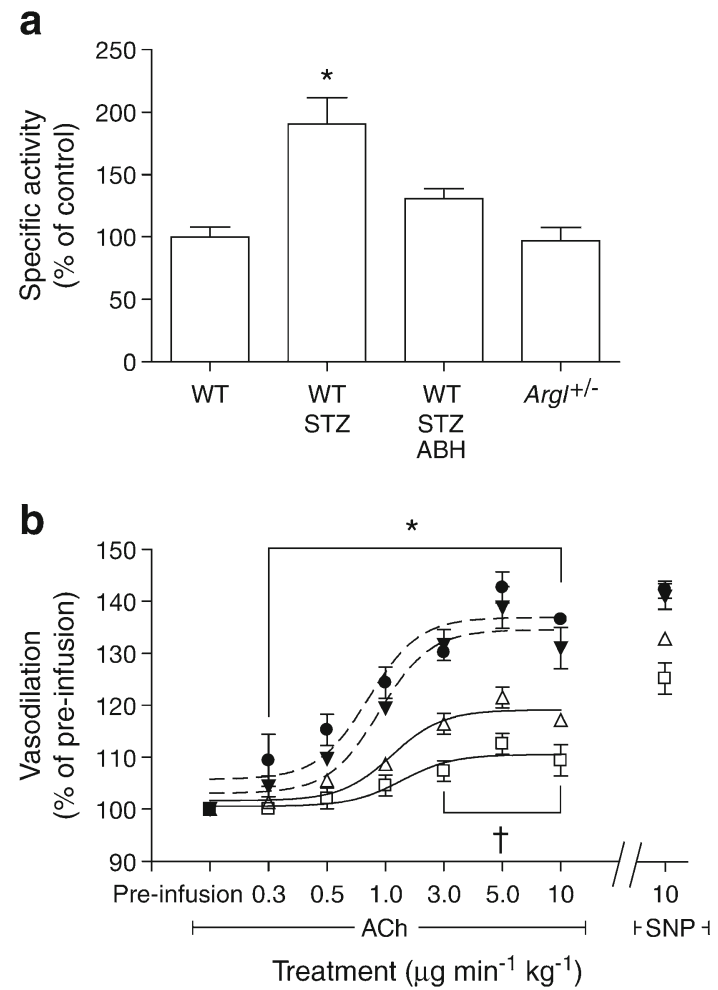

Fig. 6 Treatment with the specific arginase inhibitor $A B H$ reduced arginase activity and improved vasodilation in the WT STZ retinas. (a) Arginase activity was significantly reduced in the ABH-treated WT STZ retinas compared with the untreated WT STZ retinas $\left({ }^{*} p<0.05\right.$ WT STZ compared with all other groups, one-way ANOVA; WT, $n=4$; WT STZ, $n=5$; WT STZ ABH, $n=6 ; \operatorname{Arg} I^{+/-}, n=5$ ). (b) Dilation to $\mathrm{ACh}$ was significantly improved in the $\mathrm{ABH}$-treated mice compared with the WT STZ mice $\left({ }^{*} p<0.05 \mathrm{WT}\right.$ and $\operatorname{Arg} I^{+/}$compared with WT STZ; ${ }^{\dagger} p<0.05$ WT STZ ABH compared with WT STZ, two-way ANOVA; WT, $n=6$; WT STZ, $n=4$; WT STZ ABH, $n=10$; $\mathrm{ArgI}{ }^{+/}$, $n=4)$. Black circle, WT; white square, WT STZ; white triangle, WT STZ ABH; black triangle, $\operatorname{Arg} I^{+/-}$ 


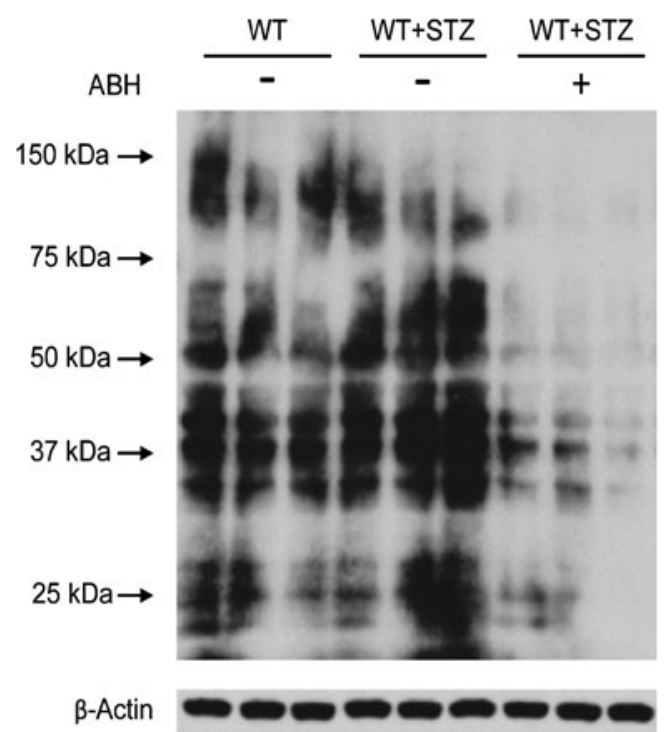

Fig. 7 Representative western blot for 3-nitrotyrosine showed greater 3-nitrotyrosine immunoreactivity in the retinas from STZ mice than those from control mice. The arginase inhibitor $\mathrm{ABH}$ blocked the formation of 3-nitrotyrosine in the STZ mice. Each experimental group consists of three lanes containing one retina per lane. $\beta$-Actin was used as the loading control

In order to assess the potential role of NOS uncoupling and $\mathrm{ONOO}^{-}$formation in diabetes-induced retinal vessel dysfunction and the role of arginase activity in this process, we used western blotting to determine the relative levels of proteins positive for 3-nitrotyrosine in the retinas of the diabetic, control and ABH-treated mice (Fig. 7). Nitration of tyrosine residues to form 3-nitrotyrosine is considered to be a marker of loss of protein function due to $\mathrm{ONOO}^{-}$formation and has been implicated in diabetic vascular disease $[12,27]$. This analysis showed that STZ treatment increased 3-nitrotyrosine immunoreactivity compared with the non-diabetic controls, and this effect was prevented by inhibiting arginase with $\mathrm{ABH}$.

Studies on central retinal arteries from rats To see whether arginase activity also has a role in retinal vascular dysfunction in diabetic rats, we performed studies using retinas and CRAs isolated from rats. Whole retinas were used for western blotting of arginase protein and arginase activity levels, and isolated CRAs were used for ex vivo analysis of vasodilation by pressure myography. The groups were controls, STZ retinas, and STZ retinas pretreated with the specific arginase inhibitor BEC $(100 \mu \mathrm{mol} / 1,45 \mathrm{~min}$ ex vivo, prior to beginning the experiments). Like $\mathrm{ABH}, \mathrm{BEC}$ is a boronic acid analogue that specifically inhibits arginase by binding as the tetrahedral boronate anion, preventing the arginine hydrolysis reaction without affecting the activity of eNOS $[14,28]$. BEC has also been used in investigations of diabetes-related reduction of corpus cavernosum endothelial function in previous ex vivo studies [29].

Retinas from diabetic rats showed increased arginase I but not arginase II protein levels compared with controls (Fig. 8a,
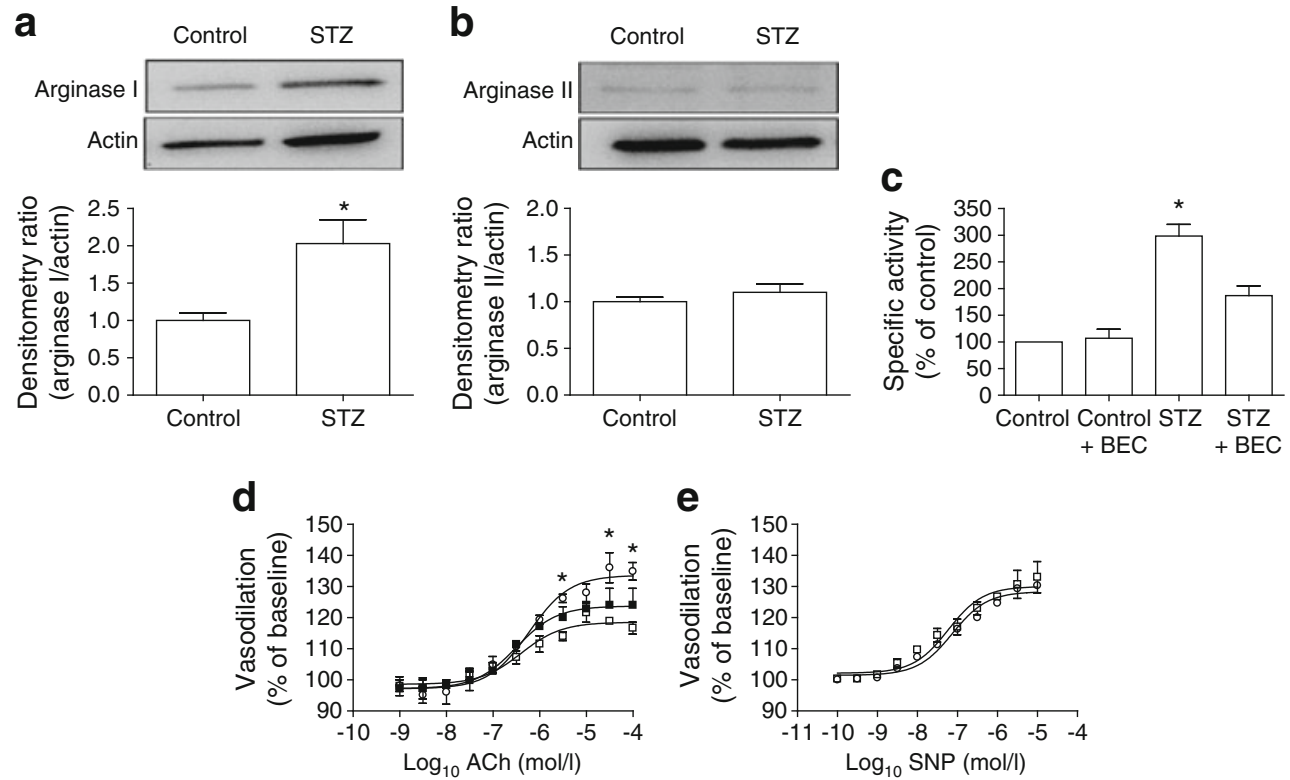

Fig. 8 Diabetes-induced increases in arginase I protein levels and arginase activity in the STZ rat retina were associated with impaired vasodilation of the CRA as shown by ex vivo pressure myography. (a, b) Western blots and densitometry show STZ-induced upregulation of $\operatorname{ArgI}$ (a) but not $\operatorname{ArgII}$ (b) compared with actin loading controls. (c) Arginase activity was significantly increased in the STZ CRAs compared with the controls $\left({ }^{*} p<0.05\right)$. This increase was significantly blunted by ex vivo treatment of the isolated vessels with the arginase inhibitor BEC $\left({ }^{*} p<0.05\right.$, one-way ANOVA; all groups, $\left.n=4\right)$. (d) Vasodilation in the CRAs from STZ rats was reduced compared with controls and with STZ vessels pretreated with BEC $\left({ }^{*} p<0.05\right.$, twoway ANOVA; all groups, $n=3$ ). (e) Dilation of CRAs to SNP was no different in the STZ diabetic rats and the age-matched controls. White circle, controls; white square, STZ; black square, STZ BEC 
b). Arginase activity was also significantly increased in diabetic retinas compared with controls and diabetic retinas pretreated ex vivo with BEC ( $p<0.05$; STZ, $298 \pm 22 \%$; control, $100 \%$; control + BEC, $106 \pm 17 \%)$. The diabetes-induced increase in arginase activity was significantly blunted in the BEC-treated retinas $(p<0.05 ; 187 \% \pm 18 \%)$. There was no significant difference between the vehicle-treated and BECtreated control retinas (Fig. 8c).

Analysis of vessel function using pressure myography involved the addition of the thromboxane analogue U-46619 $\left(3 \times 10^{-7} \mathrm{~mol} / \mathrm{l}\right)$ to the vessel chamber. This caused the vessels to constrict, with a resultant decrease in their inner diameter that was not statistically different between groups. The addition of cumulative concentrations of ACh caused a progressive increase in endothelium-dependent vasodilation. The CRA vessels from non-diabetic control rats dilated to $135 \%$ of baseline with $\mathrm{ACh}$ at the highest doses compared with $117 \%$ in the CRAs from STZ retinas $(p<0.05)$. BEC pretreatment ex vivo partially restored the ACh-induced vasodilation of the STZ CRAs to $124 \%$ of baseline (Fig. 8d). In the presence of the NOS inhibitor, L-NAME, there was no dilation to $\mathrm{ACh}$, thus indicating that dilation was an NO-dependent activity (data not shown). To determine whether the CRAs had fully functioning smooth muscle cells, SNP was utilised. There was no significant difference between control and diabetic arteries in their vasodilation to SNP (Fig. 8e).

\section{Discussion}

Decreased retinal blood flow is an early abnormality in diabetic humans and animals [1-3]. A variety of signalling pathways have been implicated in diabetes-induced vascular dysfunction, including protein kinase $\mathrm{C}$, endothelin, angiotensin II and NO. In the present study, we provide evidence that diabetes-induced impairment of endothelial-dependent vasodilation responses in the retinal vessels of both mice and rats is associated with activation of arginase along with an increase in the production of arginase I. Furthermore, the increase in arginase activity and the decrease in vasodilation to ACh are largely prevented by deletion of one copy of the arginase I gene in mice or inhibition of arginase activity in mice or rats. Previous work with other tissues and disease models has strongly implicated arginase in diabetes and oxidative-stress-induced vascular dysfunction $[14,18,19$, 26, 30]. However, our results are the first we know of to show a role for arginase I in diabetes-induced retinal vascular dysfunction.

Previous studies have demonstrated the feasibility of using microscopic imaging systems for the real-time analysis of retinal vascular function in rats [31-33] and mice [34-37]. Our experiments used a high-resolution fundus microscope system to focus on the primary branches of the
CRA in living mice while delivering vasoactive compounds systemically. This analysis showed that diabetes caused a significant impairment of endothelial-dependent vascular dilation without causing a change in basal arterial diameter, blood pressure or HR. Similarly to previously published results relating to other vessel beds, we found a diabetesinduced decrease of approximately $30-35 \%$ in the maximal response of retinal vessels to ACh [18]. This decrease was largely prevented in the knockout mouse lacking one copy of the arginase I gene.

The diabetes-induced decrease in EC-dependent vasodilation was also blunted by treatment with specific arginase inhibitors. In vivo mouse studies showed a reduction in arginase activity and an improvement in retinal vasodilation with a specific arginase inhibitor. Similar beneficial effects of inhibiting arginase were observed in experiments using pressure myography on CRAs that had been isolated from diabetic rats and then pretreated ex vivo with an arginase inhibitor. The consistency between the in vivo imaging results in mice and the ex vivo studies in rats confirms the validity of the assays, as well as the biological significance of the observations. The fact that similar results were obtained in studies using knockout mice as well as mice and rats treated with highly specific arginase inhibitors provides assurance of the specificity of the findings. These data suggest that diabetes-induced increases in arginase I expression play a primary role in the impairment of endothelial-dependent vasodilation responses and underscore the potential importance of arginase as a new therapeutic target for DR.

Previous research on potential mediators of diabetesinduced impairment of retinal blood flow has shown a significant role for activation of protein kinase $\mathrm{C}$ (PKC) and angiotensin II in altering retinal vasodilation responses [2, 31]. Activation of both the PKC and angiotensin II pathways not only leads to increases in reactive oxygen species, but has also been shown to increase arginase activity in vascular tissue [26, 30]. In addition, C-reactive protein has been shown to inhibit the endothelium-dependent dilation of retinal arteries [38]. C-reactive protein can activate RhoA/Rhokinase signalling in ECs, and RhoA/Rho-kinase is an upstream regulator of arginase [26, 39]. Thus, it is likely that arginase represents a final common pathway linking multiple mediators of vascular injury in the diabetic retina.

The lack of bioavailable NO can occur as a result of decreased production of $\mathrm{NO}$, but can also result from increased levels of superoxide, which reacts with $\mathrm{NO}$ to form $\mathrm{ONOO}^{-}$. $\mathrm{ONOO}^{-}$is a reactive nitrogen species that can directly act on the vasculature to prevent vasodilation [40]. We were able to show increased levels of protein tyrosine nitration in retinas from the STZ diabetic mice, and inhibition of this protein modification by treatment with an arginase inhibitor. The increase in nitrotyrosine formation is a key indicator of increased oxidant stress associated with 
diabetes. Importantly, the reduction in tyrosine nitration with $\mathrm{ABH}$ treatment links arginase to the increase in $\mathrm{ONOO}^{-}$, presumably by uncoupling eNOS.

Because of its similar activity to arginase I, arginase II cannot be ruled out as a player in this mechanism and is the subject of ongoing study in our laboratory. The present study was prompted by other work implicating arginase I in diabetic vascular dysfunction [18]. We used mice lacking one copy of the gene encoding arginase I, rather than homozygous knockout mice, for these studies because arginase I function in the liver is required for removal of the nitrogenous waste produced during protein metabolism. Deletion of both copies of arginase I is lethal at approximately 10 days after birth due to hyperammonaemia and encephalopathy [41]. Future studies using tissue-specific arginase I knockout mice are planned in order to avoid the toxicity associated with lack of the enzyme in the liver and to determine whether a total deletion of arginase I in the ECs can further abrogate the diabetes-induced vascular dysfunction.

A potential limitation of this work relates to the use of mouse retinal arterioles for the in vivo analysis and rat CRAs for the ex vivo analysis. We used two approaches in two species because we were unable to make direct comparisons of the different vessels in the same species or of the same vessel in different species due to the technical limitations of our methods. The mouse CRA artery is too small for pressure myography, whereas the optics of our Micron III fundus microscope system are not suitable for rats. Nevertheless, we believe that our data, showing similar functional deficits in mouse retinal arterioles and rat CRAs, provide strong support for our conclusions. Moreover, the use of the pressure myograph complements the in vivo studies and allows us to rule out any indirect effects of ACh on the systemic circulation.

Further study is needed to determine the specific cell type (s) involved in the detrimental effects of arginase activity on retinal vascular function. Results with isolated vessels suggest that the effect is mainly on vascular cells, but it is possible that arginase I production in monocytes/macrophages could also contribute to the pathology. Use of mice lacking arginase in ECs, smooth muscle cells or monocytederived cells will help to answer this question.

One of the metabolites of arginase activity is ornithine. Ornithine can be further metabolised into polyamines and proline, which are necessary for cell proliferation and collagen synthesis, respectively. Increases in their levels have been linked to vascular remodelling and stiffness [14, 42]. In a related study, rabbits fed a high-cholesterol diet were found to have increased arginase expression in the vasculature and increased remodelling [43]. This stiffness and increased intimal thickness can participate in vascular dysfunction. However, vessel diameters were not altered in our diabetic mice.
In conclusion, we have shown that arginase I is a potential therapeutic target for the treatment of DR using two species, in vivo and ex vivo methodologies and two arginase inhibitors. Whereas arginase is a protein of increasing interest in endothelial dysfunction, this is the first time it has been mechanistically linked to DR. In addition to the mechanistic findings, we have also provided a new technique for non-invasive study of the retinal vasculature. The cellular complexity of the retina makes it difficult to draw specific conclusions in an ex vivo setting. By addressing the endpoint reactivity of the retinal vasculature in vivo, we have been able to determine what the likely behaviour is in this disease. Our hope is that this offers an improved strategy for studying retinal vascular function.

Funding This research was supported by the NIH (R01-EY11766, R01-EY04618, R01-HL70215), AHA, VA Merit Review and the GHSU Vision Discovery Institute.

Duality of interest The authors declare that there is no duality of interest associated with this manuscript.

Contribution statement SCE, HAT, RWC and RBC conceived and designed the experiments. SCE, HAT, MR and ZX carried out the experiments and collected, analysed and interpreted the data. SCE and HAT drafted the article. MR, ZX, RWC and RBC critically revised the article. All authors approved the final submitted version of the manuscript.

\section{References}

1. Curtis TM, Gardiner TA, Stitt AW (2009) Microvascular lesions of diabetic retinopathy: clues towards understanding pathogenesis? Eye (Lond) 23:1496-1508

2. Clermont AC, Bursell SE (2007) Retinal blood flow in diabetes. Microcirculation 14:49-61

3. Nagaoka T, Sato E, Takahashi A, Yokota H, Sogawa K, Yoshida A (2010) Impaired retinal circulation in patients with type 2 diabetes mellitus: retinal laser Doppler velocimetry study. Invest Ophthalmol Vis Sci 51:6729-6734

4. De Caterina R, Libby P, Peng HB et al (1995) Nitric oxide decreases cytokine-induced endothelial activation. Nitric oxide selectively reduces endothelial expression of adhesion molecules and proinflammatory cytokines. J Clin Invest 96:60-68

5. Furchgott RF, Zawadzki JV (1980) The obligatory role of endothelial cells in the relaxation of arterial smooth muscle by acetylcholine. Nature 288:373-376

6. Gruetter CA, Barry BK, McNamara DB, Gruetter DY, Kadowitz PJ, Ignarro L (1979) Relaxation of bovine coronary artery and activation of coronary arterial guanylate cyclase by nitric oxide, nitroprusside and a carcinogenic nitrosoamine. J Cyclic Nucl Res 5:211-224

7. Cooke JP, Rossitch E Jr, Andon NA, Loscalzo J, Dzau VJ (1991) Flow activates an endothelial potassium channel to release an endogenous nitrovasodilator. J Clin Invest 88:1663-1671

8. Cohen RA, Tong X (2010) Vascular oxidative stress: the common link in hypertensive and diabetic vascular disease. J Cardiovasc Pharmacol 55:308-316 
9. Guzik TJ, Mussa S, Gastaldi D et al (2002) Mechanisms of increased vascular superoxide production in human diabetes mellitus: role of $\mathrm{NAD}(\mathrm{P}) \mathrm{H}$ oxidase and endothelial nitric oxide synthase. Circulation 105:1656-1662

10. Lopez-Lopez JG, Moral-Sanz J, Frazziano G et al (2008) Diabetes induces pulmonary artery endothelial dysfunction by NADPH oxidase induction. Am J Physiol Lung Cell Mol Physiol 295: L727-L732

11. Xia Y, Zweier JL (1997) Superoxide and peroxynitrite generation from inducible nitric oxide synthase in macrophages. Proc Natl Acad Sci USA 94:6954-6958

12. Pacher P, Obrosova IG, Mabley JG, Szabo C (2005) Role of nitrosative stress and peroxynitrite in the pathogenesis of diabetic complications. Emerging new therapeutical strategies. Curr Med Chem 12:267-275

13. Hein TW, Zhang C, Wang W, Chang CI, Thengchaisri N, Kuo L (2003) Ischemia-reperfusion selectively impairs nitric oxidemediated dilation in coronary arterioles: counteracting role of arginase. FASEB J 17:2328-2330

14. Berkowitz DE, White R, Li D et al (2003) Arginase reciprocally regulates nitric oxide synthase activity and contributes to endothelial dysfunction in aging blood vessels. Circulation 108:2000-2006

15. Johnson FK, Johnson RA, Peyton KJ, Durante W (2005) Arginase inhibition restores arteriolar endothelial function in Dahl rats with salt-induced hypertension. Am J Physiol Regul Integr Comp Physiol 288:R1057-R1062

16. Santhanam L, Christianson DW, Nyhan D, Berkowitz DE (2008) Arginase and vascular aging. J Appl Physiol 105:1632-1642

17. Zhang C, Hein TW, Wang W et al (2004) Upregulation of vascular arginase in hypertension decreases nitric oxide-mediated dilation of coronary arterioles. Hypertension 44:935-943

18. Romero MJ, Iddings JA, Platt DH et al (2012) Diabetes-induced vascular dysfunction involves arginase I. Am J Physiol Heart Circ Physiol 302:H159-H166

19. Romero MJ, Platt DH, Tawfik HE et al (2008) Diabetes-induced coronary vascular dysfunction involves increased arginase activity. Circ Res 102:95-102

20. Closs EI, Scheld JS, Sharafi M, Forstermann U (2000) Substrate supply for nitric-oxide synthase in macrophages and endothelial cells: role of cationic amino acid transporters. Mol Pharmacol 57:68-74

21. Topal G, Brunet A, Walch L, Boucher JL, David-Dufilho M (2006) Mitochondrial arginase II modulates nitric-oxide synthesis through nonfreely exchangeable L-arginine pools in human endothelial cells. J Pharmacol Exp Ther 318:1368-1374

22. Wu G, Morris SM Jr (1998) Arginine metabolism: nitric oxide and beyond. Biochem J 336(Pt 1):1-17

23. Zhang W, Baban B, Rojas M et al (2009) Arginase activity mediates retinal inflammation in endotoxin-induced uveitis. Am J Pathol 175:891-902

24. Baggio R, Emig FA, Christianson DW, Ash DE, Chakder S, Rattan S (1999) Biochemical and functional profile of a newly developed potent and isozyme-selective arginase inhibitor. J Pharmacol Exp Ther 290:1409-1416

25. Cox JD, Kim NN, Traish AM, Christianson DW (1999) Arginaseboronic acid complex highlights a physiological role in erectile function. Nat Struct Biol 6:1043-1047

26. Shatanawi A, Romero MJ, Iddings JA et al (2011) Angiotensin IIinduced vascular endothelial dysfunction through RhoA/Rho kinase/p38 mitogen-activated protein kinase/arginase pathway. Am J Physiol Cell Physiol 300:C1181-C1192

27. Mohiuddin I, Chai H, Lin PH, Lumsden AB, Yao Q, Chen C (2006) Nitrotyrosine and chlorotyrosine: clinical significance and biological functions in the vascular system. J Surg Res 133:143149

28. Colleluori DM, Ash DE (2001) Classical and slow-binding inhibitors of human type II arginase. Biochemistry 40:9356-9362

29. Toque HA, Tostes RC, Yao L et al (2011) Arginase II deletion increases corpora cavernosa relaxation in diabetic mice. J Sex Med $8: 722-733$

30. Chandra S, Romero MJ, Shatanawi A, Alkilany AM, Caldwell RB, Caldwell RW (2012) Oxidative species increase arginase activity in endothelial cells through the RhoA/Rho kinase pathway. Br J Pharmacol 165:506-519

31. Horio N, Clermont AC, Abiko A et al (2004) Angiotensin AT(1) receptor antagonism normalizes retinal blood flow and acetylcholine-induced vasodilatation in normotensive diabetic rats. Diabetologia 47:113-123

32. Noguchi M, Mori A, Sakamoto K, Nakahara T, Ishii K (2009) Vasodilator effects of flunarizine on retinal blood vessels in anesthetized rats. Biol Pharm Bull 32:2068-2071

33. Mori A, Saigo O, Hanada M, Nakahara T, Ishii K (2009) Hyperglycemia accelerates impairment of vasodilator responses to acetylcholine of retinal blood vessels in rats. J Pharmacol Sci 110:160 168

34. Wright WS, Harris NR (2008) Ozagrel attenuates early streptozotocin-induced constriction of arterioles in the mouse retina. Exp Eye Res 86:528-536

35. Wang Z, Yadav AS, Leskova W, Harris NR (2010) Attenuation of streptozotocin-induced microvascular changes in the mouse retina with the endothelin receptor A antagonist atrasentan. Exp Eye Res 91:670-675

36. Xu H, Manivannan A, Goatman KA et al (2002) Improved leukocyte tracking in mouse retinal and choroidal circulation. Exp Eye Res 74:403-410

37. Iwama D, Miyahara S, Tamura H, Miyamoto K, Hirose F, Yoshimura N (2008) Lack of inducible nitric oxide synthases attenuates leukocyte-endothelial cell interactions in retinal microcirculation. Br J Ophthalmol 92:694-698

38. Nagaoka T, Kuo L, Ren Y, Yoshida A, Hein TW (2008) C-reactive protein inhibits endothelium-dependent nitric oxide-mediated dilation of retinal arterioles via enhanced superoxide production. Invest Ophthalmol Vis Sci 49:2053-2060

39. Nakakuki T, Ito M, Iwasaki $\mathrm{H}$ et al (2005) Rho/Rho-kinase pathway contributes to $\mathrm{C}$-reactive protein-induced plasminogen activator inhibitor-1 expression in endothelial cells. Arterioscler Thromb Vasc Biol 25:2088-2093

40. Beckmann JS, Ye YZ, Anderson PG et al (1994) Extensive nitration of protein tyrosines in human atherosclerosis detected by immunohistochemistry. Biol Chem Hoppe Seyler 375:81-88

41. Iyer RK, Yoo PK, Kern RM et al (2002) Mouse model for human arginase deficiency. Mol Cell Biol 22:4491-4498

42. Ryoo S, Berkowitz DE, Lim HK (2011) Endothelial arginase II and atherosclerosis. Korean J Anesthesiol 61:3-11

43. Hayashi T, Esaki T, Sumi D, Mukherjee T, Iguchi A, Chaudhuri G (2006) Modulating role of estradiol on arginase II expression in hyperlipidemic rabbits as an atheroprotective mechanism. Proc Natl Acad Sci USA 103:10485-10490 